\title{
Cardiovascular risk in bus drivers
}

\author{
Chloe KY Cheung ${ }^{1}$, Sunny SL Tsang ${ }^{1}$, Oliver $\mathrm{Ho}^{1}$, Nathan Lam ${ }^{1}$, Edmund CL Lam ${ }^{1}$, Carolyn Ng$^{1}$, \\ Frances Sun ${ }^{1}$, Brian $\mathrm{Yu}^{1}$, Natalie Kwan ${ }^{1}$, Gilberto KK Leung ${ }^{1,2}$ *, MB, BS, PhD \\ ${ }^{1}$ Li Ka Shing Faculty of Medicine, The University of Hong Kong, Hong Kong \\ ${ }^{2}$ Department of Surgery, Li Ka Shing Faculty of Medicine, The University of Hong Kong, Hong Kong \\ *Corresponding author: gilberto@hku.hk
}

Hong Kong Med J 2020;26:451-6

https://doi.org/10.12809/hkmj198087

\section{Introduction}

Driver health is an important issue worldwide. Cardiovascular risk is well known to be higher in bus drivers than in the general population. ${ }^{1-5}$ Although much media attention has been drawn towards the recent increase in bus accidents in Hong Kong, ${ }^{6-8}$ few studies or measures have been carried out. The aim of this study was to examine available findings on cardiovascular risks among bus drivers and related policies on risk modification internationally and in Hong Kong.

\section{Cardiovascular risk in bus drivers}

Previous studies have documented cardiovascular risk in professional drivers. In Korea, a crosssectional study on 443 male bus drivers compared their incidence of hypertension (53.3\%) with two control groups $(17.6 \%$ and $19.7 \%$, respectively). The results showed that the odds ratio of developing a cardiovascular event in bus drivers was 2.18 to $2.58 .^{9}$ Another study was conducted through questionnaires on a randomly selected cohort of 440 professional drivers in Sweden. It found a 1.13-times higher relative risk of developing cardiovascular outcomes like stroke, and the odds ratio of developing a cardiovascular event was $2.34 .^{3}$ Before the implementation of the Labour Standards Act, studies conducted on 2297 Taiwanese bus drivers and skilled workers also showed higher incidence of hypertension in bus drivers $(72.4 \%$, 986/1361) than skilled workers $(30.6 \%, 164 / 536){ }^{2}$ The overall findings indicate that bus drivers of different nationalities exhibit a higher cardiovascular risk.

\section{Risk factors in bus drivers}

There are three main mechanisms predisposing bus drivers to higher cardiovascular risk. ${ }^{9}$ An acute episode due to a busy road with excessive traffic and aggressive drivers, is the first major cause. This is exacerbated by poor working conditions-a combination of long working hours and insufficient breaks in between shifts. These occupational factors lead to obesity, ${ }^{10}$ high blood pressure, ${ }^{11}$ and poorly controlled cholesterol levels. ${ }^{12}$ Finally, job stress from being chronically overworked is also a contributing cause. ${ }^{13}$

\section{Regulatory measures}

Regulations aimed at reducing bus drivers' cardiovascular risk are well established in Singapore, Taiwan, and South Korea. In Singapore, all transport operators must comply with the Employment Act, which limits drivers' working hours to 12 hours per day (including overtime). ${ }^{14}$ The maximum penalty for violation of regulations is SGD 5000 and a custodial sentence of 12 months. ${ }^{15}$ Under Singapore's 'Healthier Workers, Happier Workers' programme, free health talks and regular health screening are provided at bus terminals during shift changes. ${ }^{16}$ The Taiwanese Labour Standards Act sets the maximum working hours of bus drivers to 12 hours a day and uses digital tachograph for monitoring. ${ }^{17}$ In South Korea, non-compliant bus companies will be subjected to a fine or temporary suspension of service. ${ }^{9,18}$ The installation of digital tachographs to record information about driving time and rest periods of drivers also effectively monitors overwork. ${ }^{18}$

\section{Workers' compensation}

Cardiovascular risk in bus drivers is one of the most compensable conditions internationally, ${ }^{18}$ but it is not recognised in Hong Kong. In Japan, workers can be compensated for overwork-related health problems, known colloquially as 'karoshi' (death by overwork). Similarly, in Korea, the Ministry of Employment and Labor outlined criteria for work-related diseases liable for compensation to include cerebral infarction, hypertensive encephalopathy, coronary heart disease, and haemorrhagic stroke associated with existing hypertension. ${ }^{18}$ In contrast, Hong Kong bus drivers are rarely compensated, if at all, in this regard. According to the Employees' Compensation Ordinance, the Labour Department would consider whether there is a direct causal relationship between the disease and certain type of work, taking into account the availability of supportive scientific evidence. ${ }^{19}$ Cardiovascular events do not fall under the definition of occupational diseases as they can be due to other factors that have no direct relationship with work such as eating habits. 
TABLE I. Demographic of bus drivers and non-bus drivers*

\begin{tabular}{|c|c|c|c|c|}
\hline & Bus drivers $(n=255)$ & Non-bus drivers $(n=252)$ & Total $(n=507)$ & $\mathbf{P}$ valuet \\
\hline Age & $48 \pm 11$ & $52 \pm 15$ & $50 \pm 13$ & \\
\hline Age range & & & & $<0.001$ \\
\hline $25-49$ years & $127(49.8 \%)$ & $101(40.1 \%)$ & $228(45.0 \%)$ & \\
\hline $50-59$ years & $84(32.9 \%)$ & $61(24.2 \%)$ & $145(28.6 \%)$ & \\
\hline $60-84$ years & 44 (17.3\%) & 90 (35.7\%) & 134 (26.4\%) & \\
\hline \multicolumn{5}{|l|}{ Sex } \\
\hline Male & $255(100.0 \%)$ & $252(100 \%)$ & $507(100 \%)$ & \\
\hline Female & 0 & 0 & 0 & \\
\hline Angina, myocardial infarction, or strokeł & $\mathrm{n}=239$ & $\mathrm{n}=243$ & $\mathrm{n}=482$ & $<0.001$ \\
\hline Yes & $3(1.3 \%)$ & $19(7.8 \%)$ & $22(4.6 \%)$ & \\
\hline No & $236(98.7 \%)$ & $224(92.2 \%)$ & $460(95.4 \%)$ & \\
\hline Smoking status & & & & $<0.001$ \\
\hline Non-smoker & $113(44.3 \%)$ & $163(64.7 \%)$ & $276(54.4 \%)$ & \\
\hline Ex-smoker & $21(8.2 \%)$ & $38(15.1 \%)$ & $59(11.6 \%)$ & \\
\hline Light smoker ( $<10$ cigarettes per day) & $46(18.0 \%)$ & $22(8.7 \%)$ & $68(13.4 \%)$ & \\
\hline Moderate smoker (10-19 cigarettes per day) & $50(19.6 \%)$ & $19(7.5 \%)$ & $69(13.6 \%)$ & \\
\hline Heavy smoker (>20 cigarettes per day) & $25(9.8 \%)$ & $10(4.0 \%)$ & $35(6.9 \%)$ & \\
\hline Diabetes status & & & & 0.049 \\
\hline None & $218(85.5 \%)$ & $208(82.5 \%)$ & $426(84.0 \%)$ & \\
\hline Type 1 & $1(0.4 \%)$ & 0 & $1(0.2 \%)$ & \\
\hline Type 2 & $36(14.1 \%)$ & $44(17.5 \%)$ & $80(15.8 \%)$ & \\
\hline $\begin{array}{l}\text { Angina or heart attack in a first-degree relative } \\
\text { aged }<60 \text { years }\end{array}$ & & & & $<0.001$ \\
\hline Yes & $37(14.5 \%)$ & $55(21.8 \%)$ & $92(18.1 \%)$ & \\
\hline No & $218(85.5 \%)$ & $197(78.2 \%)$ & 415 (81.9\%) & \\
\hline Chronic kidney disease (stage $3,4,5$ ) & & & & $<0.001$ \\
\hline Yes & 0 & $3(1.2 \%)$ & $3(0.6 \%)$ & \\
\hline No & $255(100 \%)$ & $249(98.8 \%)$ & $504(99.4 \%)$ & \\
\hline Atrial fibrillation & & & & $<0.001$ \\
\hline Yes & $1(0.4 \%)$ & $8(3.2 \%)$ & $9(1.8 \%)$ & \\
\hline No & $254(99.6 \%)$ & $244(96.8 \%)$ & $498(98.2 \%)$ & \\
\hline Receiving blood pressure treatment & & & & 0.075 \\
\hline Yes & $61(23.9 \%)$ & $69(27.4 \%)$ & $130(25.6 \%)$ & \\
\hline No & $194(76.1 \%)$ & $183(72.6 \%)$ & 377 (74.4\%) & \\
\hline Migraine & & & & 0.655 \\
\hline Yes & $14(5.5 \%)$ & $15(6.0 \%)$ & $29(5.7 \%)$ & \\
\hline No & $241(94.5 \%)$ & 237 (94.0\%) & $478(94.3 \%)$ & \\
\hline Rheumatoid arthritis & & & & 0.042 \\
\hline Yes & $1(0.4 \%)$ & $3(1.2 \%)$ & $4(0.8 \%)$ & \\
\hline No & $254(99.6 \%)$ & 249 (98.8\%) & $503(99.2 \%)$ & \\
\hline \multicolumn{5}{|l|}{ Systemic lupus erythematosus } \\
\hline Yes & 0 & 0 & 0 & \\
\hline No & $255(100 \%)$ & $252(100 \%)$ & $507(100 \%)$ & \\
\hline Severe mental illness & & & & 0.044 \\
\hline Yes & 0 & $1(0.4 \%)$ & $1(0.2 \%)$ & \\
\hline No & $255(100 \%)$ & $251(99.6 \%)$ & $506(99.8 \%)$ & \\
\hline Receiving atypical antipsychotic medication & & & & 0.006 \\
\hline Yes & $1(0.4 \%)$ & $4(1.6 \%)$ & $5(1.0 \%)$ & \\
\hline No & $254(99.6 \%)$ & $248(98.4 \%)$ & $502(99.0 \%)$ & \\
\hline Receiving regular steroids & & & & 0.216 \\
\hline Yes & $6(2.4 \%)$ & $4(1.6 \%)$ & $10(2.0 \%)$ & \\
\hline No & $249(97.6 \%)$ & $248(98.4 \%)$ & $497(98.0 \%)$ & \\
\hline
\end{tabular}

Abbreviation: QRISK3 = cardiovascular risk algorithm version 3 calculator

* Data are presented as mean \pm standard deviation or No. (\%) of patients, unless otherwise specified

$+\mathrm{P}$ value was tested by independent $t$ test

‡ Data missing because items were not reported in the questionnaire 
TABLE I. (cont'd)

\begin{tabular}{|c|c|c|c|c|}
\hline & Bus drivers $(n=255)$ & Non-bus drivers $(n=252)$ & Total $(n=507)$ & $\mathbf{P}$ value \\
\hline Erectile dysfunction & & & & 0.044 \\
\hline Yes & 0 & $1(0.4 \%)$ & $1(0.2 \%)$ & \\
\hline No & $255(100 \%)$ & 251 (99.6\%) & $506(99.8 \%)$ & \\
\hline Systolic blood pressureł & $n=253$ & $\mathrm{n}=251$ & $\mathrm{n}=504$ & 0.001 \\
\hline$<120 \mathrm{~mm} \mathrm{Hg}$ & $22(8.7 \%)$ & $41(16.3 \%)$ & $63(12.5 \%)$ & \\
\hline $120-129 \mathrm{~mm} \mathrm{Hg}$ & $72(28.5 \%)$ & $81(32.3 \%)$ & $153(30.4 \%)$ & \\
\hline$>130 \mathrm{~mm} \mathrm{Hg}$ & $159(62.8 \%)$ & $129(51.4 \%)$ & $288(57.1 \%)$ & \\
\hline Hours worked per day $\ddagger$ & $\mathrm{n}=255$ & $\mathrm{n}=226$ & $\mathrm{n}=481$ & $<0.001$ \\
\hline 0-4 Hours & 0 & $23(10.2 \%)$ & $23(4.8 \%)$ & \\
\hline 5-9 Hours & $35(13.7 \%)$ & $142(62.8 \%)$ & $177(36.8 \%)$ & \\
\hline 10-14 Hours & $220(86.3 \%)$ & $61(27.0 \%)$ & $281(58.4 \%)$ & \\
\hline Hours worked per shift & $\mathrm{n}=253$ & $n=133$ & $\mathrm{n}=386$ & 0.069 \\
\hline 0-1 Hours & $2(0.8 \%)$ & $4(3.0 \%)$ & $6(1.6 \%)$ & \\
\hline 1-2 Hours & $7(2.8 \%)$ & $5(3.8 \%)$ & $12(3.1 \%)$ & \\
\hline 2-3 Hours & $1(0.4 \%)$ & $14(10.5 \%)$ & $15(3.9 \%)$ & \\
\hline 3-4 Hours & $21(8.3 \%)$ & $43(32.3 \%)$ & $64(16.6 \%)$ & \\
\hline 4-5 Hours & $24(9.5 \%)$ & $35(26.3 \%)$ & $59(15.3 \%)$ & \\
\hline 5-6 Hours & $29(11.5 \%)$ & $10(7.5 \%)$ & $39(10.1 \%)$ & \\
\hline$>6$ Hours & $169(66.8 \%)$ & $22(16.5 \%)$ & $191(49.5 \%)$ & \\
\hline Hours of exercise per day $\ddagger$ & $\mathrm{n}=255$ & $\mathrm{n}=251$ & $\mathrm{n}=506$ & $<0.001$ \\
\hline$<0.5$ Hours & $158(62.0 \%)$ & $94(37.5 \%)$ & $252(49.8 \%)$ & \\
\hline 0.5-1 Hours & $77(30.2 \%)$ & $86(34.3 \%)$ & $163(32.2 \%)$ & \\
\hline 1-1.5 Hours & $15(5.9 \%)$ & $44(17.5 \%)$ & $59(11.7 \%)$ & \\
\hline 1.5-2 Hours & $3(1.2 \%)$ & $15(6.0 \%)$ & $18(3.6 \%)$ & \\
\hline$>2$ Hours & $2(0.8 \%)$ & $12(4.8 \%)$ & $14(2.8 \%)$ & \\
\hline Angina & & & & 0.004 \\
\hline Yes & $14(5.5 \%)$ & $22(8.7 \%)$ & $36(7.1 \%)$ & \\
\hline No & $241(94.5 \%)$ & $230(91.3 \%)$ & $471(92.9 \%)$ & \\
\hline Palpitations & & & & $<0.001$ \\
\hline Yes & $16(6.3 \%)$ & $31(12.3 \%)$ & 47 (9.3\%) & \\
\hline No & $239(93.7 \%)$ & $221(87.7 \%)$ & $460(90.7 \%)$ & \\
\hline Dizziness & & & & 0.001 \\
\hline Yes & $45(17.6 \%)$ & $59(23.4 \%)$ & $104(20.5 \%)$ & \\
\hline No & $210(82.4 \%)$ & $193(76.6 \%)$ & $403(79.5 \%)$ & \\
\hline Shortness of breath & & & & 0.003 \\
\hline Yes & 9 (3.5\%) & $16(6.3 \%)$ & $25(4.9 \%)$ & \\
\hline No & $246(96.5 \%)$ & $236(93.7 \%)$ & $482(95.1 \%)$ & \\
\hline Body mass index & & & & 0.225 \\
\hline$<18.5$ & $14(5.5 \%)$ & $13(5.2 \%)$ & $27(5.3 \%)$ & \\
\hline $18.5-24.9$ & $172(67.5 \%)$ & $168(66.7 \%)$ & $340(67.1 \%)$ & \\
\hline $25-29.9$ & $62(24.3 \%)$ & $63(25.0 \%)$ & $125(24.7 \%)$ & \\
\hline$\geq 30$ & $7(2.7 \%)$ & $8(3.2 \%)$ & $15(3.0 \%)$ & \\
\hline QRISK3 score & & & & $<0.001$ \\
\hline$<10$ & $206(80.8 \%)$ & $169(67.1 \%)$ & $375(74.0 \%)$ & \\
\hline $10-20$ & $37(14.5 \%)$ & $46(18.3 \%)$ & $83(16.4 \%)$ & \\
\hline$>20$ & $12(4.7 \%)$ & $37(14.7 \%)$ & $49(9.7 \%)$ & \\
\hline Healthy score & & & & $<0.001$ \\
\hline$<10$ & $248(97.3 \%)$ & $212(84.1 \%)$ & $460(90.7 \%)$ & \\
\hline $10-20$ & $6(2.4 \%)$ & $32(12.7 \%)$ & $38(7.5 \%)$ & \\
\hline$>20$ & $1(0.4 \%)$ & $8(3.2 \%)$ & $9(1.8 \%)$ & \\
\hline Relative risk range & & & & $<0.001$ \\
\hline$<1$ & $14(5.5 \%)$ & $24(9.5 \%)$ & $38(7.5 \%)$ & \\
\hline 1 & $17(6.7 \%)$ & 25 (9.9\%) & $42(8.3 \%)$ & \\
\hline$>1$ & $224(87.8 \%)$ & $203(80.6 \%)$ & $427(84.2 \%)$ & \\
\hline Healthy heart age range & & & & $<0.001$ \\
\hline$<30.0$ Years & 0 & 0 & 0 & \\
\hline $30-60$ Years & 0 & 0 & 0 & \\
\hline$\geq 60$ Years & $255(100 \%)$ & $252(100 \%)$ & $507(100 \%)$ & \\
\hline
\end{tabular}




\section{Situation in Hong Kong}

Recently, we conducted a questionnaire survey on 255 bus drivers and 252 non-bus drivers in Hong Kong (Table 1). We included Chinese men aged 25 to 84 years; recruitment of bus drivers was done at bus terminals and non-bus drivers were approached at random outside the exits to train stations. The QRISK3 (cardiovascular risk algorithm version 3 calculator) was used to assess cardiovascular risk by quantifying the 10-year risk for myocardial infarction and stroke in adults. Established cardiovascular risk factors including hypertension, hyperlipidaemia, diabetes, and smoking, and newly identified cardiovascular risk factors were taken into account. ${ }^{20}$ Systolic blood pressure values were obtained either by the drivers' self-reporting, or by an automatic sphygmomanometer on the spot. Other parameters were based on subjects' own recall.

Our results (Table 2) showed that the relative risk of developing cardiovascular diseases in bus drivers (2.41) is higher than in non-bus drivers (1.84) after age adjustment. This was attributable to low exercise levels, poor job satisfaction, and long durations of working hours (Table 3). The odds of developing hypertension $(>140 / 90 \mathrm{~mm} \mathrm{Hg})$ in bus drivers was 1.62-times higher than in non-bus drivers (Table 4). Physical inactivity and smoking were factors predisposing to hypertension. Only $7.9 \%$ of bus drivers did physical exercise for $>1$ hour per day, and $47.4 \%$ were smokers, compared with $20.2 \%$ of non-bus drivers (Table 1 ). The sedentary lifestyle of bus drivers in Hong Kong is consistent with previous studies that indicated bus drivers spend $83 \%$ of their time at work sitting. ${ }^{21}$

\section{Recommendations for Hong Kong bus drivers}

Bus driver health is crucial for ensuring the safety of drivers, passengers, and other road-users. According to the World Health Organization recommendations, 30 minutes of aerobic exercise 3 times per week is ideal; short of that, there are still added benefits in moving from the category of "no activity" to "some levels" of activity. ${ }^{22}$ Smoking cessation can be introduced in a stepwise fashion, from counselling sessions, nicotine replacement therapy, and varenicline use, to community interventions introduced by the government. ${ }^{23}$ Educational programmes may be implemented within communities of professional drivers in order to improve the general health of bus drivers.

Currently, Hong Kong bus operators need to adhere to the Transport Department's Guidelines regarding maximum duty hours (12 hours), maximum driving hours (10 hours), and minimum rest time (40 minutes after 6 hours of driving). ${ }^{19}$ However, bus operators are only issued warning letters for non-compliance. Indeed, previous surveys have shown that $51 \%$ of Hong Kong franchised bus drivers worked $\geq 2$ hours of overtime per day. ${ }^{24}$ Therefore, there is a need for the relevant authorities to take action to safeguard bus drivers against overwork and associated health hazards. To enhance road safety, the feasibility of implementing regulatory

TABLE 2. Cardiovascular risk parameters for bus drivers and non-bus drivers stratified by age*

\begin{tabular}{|c|c|c|c|c|c|c|}
\hline Age-group & $\begin{array}{l}\text { Systolic blood } \\
\text { pressure }(\mathrm{mm} \mathrm{Hg})\end{array}$ & Body mass index & QRISK3 score & Healthy score & Relative risk & Healthy heart age \\
\hline \multicolumn{7}{|l|}{ Bus drivers } \\
\hline 25-49 Years & $125(131 \pm 11)$ & $127(23.3 \pm 3.5)$ & $127(3.31 \pm 4.90)$ & $127(1.34 \pm 2.16)$ & $127(2.79 \pm 2.97)$ & $127(47.7 \pm 12.45)$ \\
\hline 50-59 Years & $84(136 \pm 11)$ & $84(24.3 \pm 3.0)$ & $84(7.85 \pm 4.54)$ & $84(3.57 \pm 0.89)$ & $84(2.25 \pm 1.30)$ & $84(63.24 \pm 7.78)$ \\
\hline 60-84 Years & $44(133 \pm 11)$ & $44(23.4 \pm 2.7)$ & $44(10.25 \pm 5.92)$ & $44(6.28 \pm 2.12)$ & $44(1.61 \pm 0.81)$ & $44(66.3 \pm 10.7)$ \\
\hline Total & $253(133 \pm 11)$ & $255(23.7 \pm 3.2)$ & $255(6.01 \pm 5.70)$ & $255(2.93 \pm 2.58)$ & $255(2.41 \pm 2.29)$ & $255(56.05 \pm 13.63)$ \\
\hline \multicolumn{7}{|l|}{ Non-bus drivers } \\
\hline 25-49 Years & $100(126 \pm 11)$ & $101(23.4 \pm 3.5)$ & $101(2.3 \pm 3.34)$ & $101(1.3 \pm 1.90)$ & $101(2.01 \pm 1.93)$ & $101(43.57 \pm 12.48)$ \\
\hline 50-59 Years & $61(127 \pm 12)$ & $61(23.5 \pm 3.2)$ & $61(7.22 \pm 5.24)$ & $61(4.22 \pm 1.82)$ & $61(1.64 \pm 0.84)$ & $61(61.3 \pm 9.3)$ \\
\hline $60-84$ Years & $90(134 \pm 11)$ & $90(23.9 \pm 3.3)$ & $90(17.5 \pm 10.82)$ & $90(10.24 \pm 5.49)$ & $90(1.79 \pm 1.03)$ & $90(73.23 \pm 10.02)$ \\
\hline Total & $251(129 \pm 12)$ & $252(23.6 \pm 3.4)$ & $252(8.92 \pm 9.86)$ & $252(5.2 \pm 5.33)$ & $252(1.84 \pm 1.43)$ & $252(58.46 \pm 16.96)$ \\
\hline \multicolumn{7}{|l|}{ All } \\
\hline 25-49 Years & $225(129 \pm 11)$ & $228(23.4 \pm 3.5)$ & $228(2.87 \pm 4.30)$ & $228(1.32 \pm 2.05)$ & $228(2.44 \pm 2.59)$ & $228(45.89 \pm 12.61)$ \\
\hline 50-59 Years & $145(132 \pm 12)$ & $145(24 \pm 3.1)$ & $145(7.59 \pm 4.84)$ & $145(3.84 \pm 1.39)$ & $145(1.99 \pm 1.16)$ & $145(62.42 \pm 8.48)$ \\
\hline $60-84$ Years & $134(134 \pm 11)$ & $134(23.7 \pm 3.1)$ & $134(15.12 \pm 10.07)$ & $134(8.94 \pm 5.01)$ & $134(1.79 \pm 0.96)$ & $134(70.96 \pm 10.72)$ \\
\hline Total & $504(131 \pm 12)$ & $507(23.6 \pm 3.3)$ & $507(7.46 \pm 8.16)$ & $507(4.06 \pm 4.33)$ & $507(2.13 \pm 1.93)$ & $507(57.24 \pm 15.41)$ \\
\hline
\end{tabular}

Abbreviation: QRISK3 = cardiovascular risk algorithm version 3 calculator

* Data are presented as No. (mean \pm standard deviation) 
TABLE 3. Pearson correlations $\left(r^{2}\right)$ between relative risk and other variables for bus drivers and non-bus drivers

\begin{tabular}{lccc}
\hline Variable & $\begin{array}{c}\text { No. of } \\
\text { patients }\end{array}$ & $\mathbf{r}^{2}$ & P value \\
\hline Satisfaction score & 473 & $-0.174^{*}$ & $<0.001$ \\
\hline Pressure score & 473 & -0.007 & 0.875 \\
Hours worked per day & 481 & $0.149^{*}$ & 0.001 \\
Hours of exercise per day & 506 & $-0.146^{*}$ & 0.001 \\
Hours worked per shift & 386 & $0.119^{*}$ & 0.020 \\
\hline
\end{tabular}

* Correlation is significant at the 0.05 level (2-tailed)

TABLE 4. Odds ratio of cardiovascular event risk to bus driving

\begin{tabular}{lc}
\hline & $\begin{array}{c}\text { Odds ratio } \\
\text { (95\% confidence interval) }\end{array}$ \\
\hline Hypertension & $1.62(1.13-2.31)$ \\
Diabetes & $0.80(0.50-1.29)$ \\
Overweight & $0.95(0.64-1.40)$ \\
QRISK3 score $>10 \%$ & $0.48(0.32-0.73)$ \\
Angina & $0.61(0.30-1.22)$ \\
Palpitations & $0.48(0.25-0.90)$ \\
Dizziness & $0.7(0.45-1.08)$ \\
Shortness of breath & $0.54(0.23-1.24)$ \\
\hline
\end{tabular}

Abbreviation: QRISK3 = cardiovascular risk algorithm version 3 calculator

measures or installation of digital tachographs to monitor work hours and regular health screening programmes should be explored in Hong Kong.

\section{Conclusion}

There is a higher relative risk of developing cardiovascular diseases in bus drivers in Hong Kong although most exhibit few cardiovascular symptoms and belong to a younger age-group. The odds of having hypertension were significantly higher in bus drivers than in non-bus drivers. Stronger advocacy for better lifestyle habits among bus drivers is needed. Future studies should elucidate the causative relationship between bus driving and cardiovascular risk to complement existing policies on employee compensation.

\section{Author contributions}

All authors contributed to the concept of the study, acquisition and analysis of the data, drafting of the manuscript, and critical revision of the manuscript for important intellectual content. All authors had full access to the data, contributed to the study, approved the final version for publication, and take responsibility for its accuracy and integrity.

\section{Conflicts of interest}

All authors have disclosed no conflicts of interest.

\section{Acknowledgement}

We thank all the survey participants, without whose support this commentary would not have been possible.

\section{Funding/support}

This commentary received no specific grant from any funding agency in the public, commercial, or not-for-profit sectors.

\section{Ethics approval}

This study was approved by the Institutional Review Board of the University of Hong Kong/Hospital Authority Hong Kong West Cluster (Ref UW 18-340). Informed consent from subjects was obtained verbally.

\section{References}

1. Belkić K, Savić C., Theorell T, Rakić L, Ercegovac D, Djordjević M. Mechanisms of cardiac risk among professional drivers. Scand J Work Environ Health 1994;20:73-86.

2. Wang PD, Lin RS. Coronary heart disease risk factors in urban bus drivers. Public Health 2001;115:261-4.

3. Hedberg GE, Jacobsson, KA, Janlert U, Langendoen S. Risk indicators of ischemic heart disease among male professional drivers in Sweden. Scand J Work Environ Health 1993;19:326-33.

4. Tüchsen, F, Hannerz H, Roepstorff C, Krause N. Stroke among male professional drivers in Denmark, 1994-2003. Occup Environ Med 2006;63:456-60.

5. Bigert C, Gustavsson P, Hallqvist J, et al. Myocardial infarction among professional drivers. Epidemiology 2003;14:333-9.

6. South China Morning Post. Hong Kong bus drivers plan work-to-rule over Transport Department's failure to remove guidelines that can lead to 14-hour shifts. 2018. Available from: http://www.scmp.com/news/hong-kong/ community/article/2147157/hong-kong-bus-drivers-planwork-rule-over-transport. Accessed 28 May 2018.

7. South China Morning Post. Hong Kong bus drivers' working hours in question after fatal crash. 2017. Available from: http://www.scmp.com/news/hong-kong/community/ article/2113275/hong-kong-bus-drivers-working-hoursquestion-after-fatal. Accessed 28 May 2018.

8. Chinadaily.com.cn. HK needs to mandate maximum working hours. 2017. Available from: http://www.chinadaily. com.cn/hkedition/2017-09/26/content_32492186.htm. Accessed 28 May 2018.

9. Shin SY, Lee CG, Song HS, et al. Cardiovascular disease risk of bus drivers in a city of Korea. Ann Occup Environ Med 2013;25:34.

10. Rosso GL, Perotto M, Feola M, Bruno G, Caramella M. Investigating obesity among professional drivers: the high risk professional driver study. Am J Ind Med 2015;58:2129.

11. Lee JW, Lee NS, Lee KJ, Kim JJ. The association between hypertension and lifestyle in express bus drivers. Korean J Occup Environ Med 2011;23:270.

12. Catalina-Romero C, Calvo E, Sánchez-Chaparro MA, et al. The relationship between job stress and dyslipidemia. Scand J Public Health 2013;41:142-9.

13. Park J. Impact of acute coronary syndrome (ACS) on work-relatedness evaluation in cerebrovascular and cardiovascular diseases among workers. J Occup Health 
2006;48:141-4

14. Ministry of Manpower, Singapore Government. About the Employment Act. Available from: https://www.mom. gov.sg/employment-practices/employment-act. 2012. Accessed 28 May 2018.

15. Ministry of Transport, Singapore Government. Written reply by Minister for Transport Lui Tuck Yew to parliamentary question on average working hours of public transport drivers. 2012. Available from: https://www.mot. gov.sg/news-centre/news/Detail/Written\%20reply\%20 by\%20Minister\%20for\%20Transport\%20Lui\%20Tuck\%20 Yew\%20to\%20Parliamentary\%20Question\%20on\%20 Average\%20Working\%20Hours\%20of\%20Public\%20 Transport\%20Drivers/. Accessed 28 May 2018.

16. Health Promotion Board, Singapore Government. Tripartite Oversight Committee on Workplace Health: 2014-2017 Report. 2017. Available from: https://www. hpb.gov.sg/docs/default-source/default-documentlibrary/tripartite-oversight-commitee-report_fa_hires. pdf?sfvrsn=7d34f672 0. Accessed 28 May 2018.

17. 台灣交通部《車輛安全檢測基準第十六點之一修正規 定》. Available from: https://law.moj.gov.tw/LawClass/ LawAll.aspx?pcode=K0040003. Accessed 31 Jan 2018.

18. Kim DS, Kang SK. Work-related cerebro-cardiovascular diseases in Korea.J Korean Med Sci2010;25(Suppl):S105-11.
19. Hong Kong SAR Government. LCQ12: Occupational safety and health of professional drivers [press release]. 2018 Jan 17. Available from: https://www.info.gov.hk/gia/ general/201801/17/P2018011700708.htm. Accessed 28 May 2018.

20. Mueller PS. QRISK3 score estimates 10-year risk for myocardial infarction and stroke. N Engl J Med 2017;357:j2099.

21. Varela-Mato V, Yates T, Stensel DJ, Biddle SJ, Clemes SA. Time spent sitting during and outside working hours in bus drivers: a pilot study. Prev Med Rep 2015;3:36-9.

22. World Health Organization. Physical activity and adults. 2015. Available from https://www.who.int/ dietphysicalactivity/factsheet_adults/en/. Accessed 28 May 2018.

23. World Health Organization. Quitting tobacco. 2011. Available from https://www.who.int/tobacco/quitting/ summary_data/en/. Accessed 28 May 2018.

24. Legislative Council, Hong Kong SAR Government. Measures managing long working hours and occupational fatigue of bus drivers. Available from https://www. legco.gov.hk/research-publications/english/essentials1718ise07-measures-managing-long-working-hoursand-occupational-fatigue-of-bus-drivers.htm\#endnote8. Accessed 28 May 2018. 